\title{
Beyond Trial-by-Trial Adaptation: A Quantification of the Time Scale of Cognitive Control
}

\author{
Bart Aben \\ Vrije Universiteit Brussel
}

\author{
Tom Verguts \\ Ghent University
}

\author{
Eva Van den Bussche \\ Vrije Universiteit Brussel
}

\begin{abstract}
The idea that adaptation to stimulus or response conflict can operate over different time scales takes a prominent position in various theories and models of cognitive control. The mechanisms underlying temporal variations in control are nevertheless poorly understood, which is partly due to a lack of appropriate empirical measures. Inspired by reinforcement learning models, we developed a method to quantify the time scale of control behaviorally, by computing trial-by-trial effects that go beyond the preceding trial. Briefly, we extended the congruency sequence effect from 1 trial to multiple trials into the past and quantified the influence of previous trials on current-trial performance as a function of trial distance. The rate at which this influence changes across trials was taken as a measure of the time scale of control. We applied the method to a flanker task with different conflict frequencies and volatility. Results showed that the time scale of control was smaller in rare-conflict and volatile contexts, compared to frequent-conflict and neutral contexts. This is in agreement with theories differentiating transient from sustained control. The method offers new opportunities to reveal temporal differences in control modes and can easily be applied to various empirical paradigms.
\end{abstract}

Public Significance Statement

People can apply cognitive control at different time scales. However, behavioral measures of such temporal variations are lacking. Here we provide a new method to quantify the time scale of cognitive control and use it to differentiate between contexts with rare and frequent conflict. The method is easily applicable in a broad range of paradigms and opens new possibilities to characterize differences in control time scale within individuals, between individuals, and between groups.

Keywords: cognitive control, conflict, congruency sequence effect, trial-by-trial adaptation, conflict adaptation

Supplemental materials: http://dx.doi.org/10.1037/xhp0000324.supp

The ability to flexibly adjust behavior to environmental demands is crucial for efficient goal achievement. For example, when faced with conflicting response options, we need cognitive control to overcome automatic response tendencies in favor of more appropriate behavior. Several theories have suggested that cognitive control can operate on different time scales (e.g., Braver, 2012; Jiang, Heller, \& Egner, 2014; Ridderinkhof,

This article was published Online First January 12, 2017.

Bart Aben, Department of Experimental and Applied Psychology, Vrije Universiteit Brussel; Tom Verguts, Department of Experimental Psychology, Ghent University; Eva Van den Bussche, Department of Experimental and Applied Psychology, Vrije Universiteit Brussel.

Tom Verguts and Eva Van den Bussche share senior authorship.

Correspondence concerning this article should be addressed to Bart Aben, Department of Experimental and Applied Psychology, Vrije Universiteit Brussel, Pleinlaan 2, B-1050, Brussels, Belgium. E-mail: bart.aben@vub.ac.be
2002). These scales can range from a fast-changing adjustment of control on a trial-by-trial basis (e.g., Gratton, Coles, \& Donchin, 1992) to a more stable control mode that is sustained over multiple trials (e.g., Bugg \& Chanani, 2011; Jimura, Locke, \& Braver, 2010). Temporal variations are often used to explain differences in cognitive control between individuals and conditions (e.g., Appelbaum, Boehler, Davis, Won, \& Woldorff, 2014; Funes, Lupiáñez, \& Humphreys, 2010; Purmann, Badde, \& Wendt, 2009). However, the lack of a behavioral quantification of the time scale of control has hampered progress in this field.

Several measures of cognitive control have been introduced over the past decades. The congruency effect (CE), for example, reflects slower response times (RTs) and lower accuracy on conflict trials (i.e., incongruent trials), compared to trials without conflict (i.e., congruent trials). A classic example of the $\mathrm{CE}$ is the Stroop effect, where ink color and word name can be congruent (e.g., the word red printed in red) or incongruent (e.g., the word red printed in green; Stroop, 1935). 
Modulations of the CE are often used to infer the time scale of control. The CE is for example subject to trial-by-trial adaptations, meaning that conflict not only affects performance on the current trial but also on the next one. This is reflected in the congruency sequence effect (CSE), which entails a smaller CE when the preceding trial is incongruent, compared to congruent (Gratton et al., 1992; for reviews, see Duthoo, Abrahamse, Braem, Boehler, \& Notebaert, 2014; Egner, 2007). The CSE has been ascribed to conflict-driven adaptation of control (Botvinick, Braver, Barch, Carter, \& Cohen, 2001). After an incongruent trial, control is increased, which diminishes interference on the subsequent incongruent trial. A congruent trial results in a decrease of control and hence increases the vulnerability to conflict on the next trial. In a way, the CSE can be thought of as a continuation of control across two trials and in this sense as indexing anticipatory or sustained control. However, it fluctuates from trial to trial and in that sense is fast-changing or transient. In fact, it has been argued that the CSE partly results from a carryover of within-trial control adjustments, which would imply that it reflects an even shorter time scale of control (Nigbur, Schneider, Sommer, Dimigen, \& Stürmer, 2015; Scherbaum, Fischer, Dshemuchadse, \& Goschke, 2011). Regardless, the range of the CSE is small, capturing fast trial-by-trial adjustments and not variations in control over a longer time scale.

Other studies have demonstrated that the CE is not only affected by previous-trial congruency but also by trials preceding the previous trial. For example, the $\mathrm{CE}$ decreases when the number of incongruent trials in the previous three to four trial increases (Horga et al., 2011; Jiménez \& Méndez, 2013). This is in accordance with models that predict that control on the current trial results from the accumulated effect of previously encountered incongruent trials (Botvinick et al., 2001; Verguts \& Notebaert, 2008) and it shows that trial-congruency can have effects that exceed the adjacent trial.

Extending this scope even further, it is also well known that the $\mathrm{CE}$ is modulated by the general conflict context, or the proportion of (in)congruent trials within a block (Bugg \& Crump, 2012; Logan \& Zbrodoff, 1979). The proportion congruency effect (PCE) entails that the $\mathrm{CE}$ is larger in blocks with mainly congruent (MC) trials than in blocks with mainly incongruent (MI) trials. This has been interpreted as a behavioral marker for transient control in MC blocks, involving short upregulations of control in response to rare incongruent trials, and sustained control in MI blocks, involving stable control that is less subject to trial-by-trial adaptations (e.g., De Pisapia \& Braver, 2006; Kane \& Engle, 2003). Although this explanation for the PCE has been challenged by accounts referring to item-specific control (Jacoby, Lindsay, \& Hessels, 2003) and contingency learning (Schmidt \& Besner, 2008), recent findings do emphasize a role for block-wide strategic control in the PCE (e.g., Bugg, Diede, Cohen-Shikora, \& Selmeczy, 2015; Bugg, McDaniel, Scullin, \& Braver, 2011; Entel, Tzelgov, \& Bereby-Meyer, 2014; Torres-Quesada, Funes, \& Lupiáñez, 2013). The exact mechanism behind this effect is nevertheless still unclear. While the CSE is bound to the previous trial by definition, the behavioral extent of the PCE is not delineated. For example, it is not known how many trials in the past drive the PCE or how the influence of previous trials on the current trial is affected by the temporal distance between them. Hence, with the
CSE and PCE, we have two measures at our disposal that each lack sensitivity to identify variations in the time scale of control.

The evaluation of temporally different control modes is hampered by this empirical ambiguity. This is problematic because variations in time scales are assumed in several models of cognitive control. The dual mechanisms of control model, for example, explains the PCE by two control mechanisms that operate on different time scales (i.e., reactive and proactive control; Braver, 2012; De Pisapia \& Braver, 2006). Other models suggest control is adjusted based on a weighted average of conflict on all preceding trials, explaining both the CSE and PCE in a single cognitive control system (Botvinick et al., 2001; Verguts \& Notebaert, 2008). A time scale parameter then determines the relative importance of recent versus more remote trials (Botvinick et al., 2001). The Bayesian model by Jiang and colleagues (2014) in addition allows this time scale parameter to adapt as a function of trial history.

Summing up, although time scale variations are crucial in models and theories of cognitive control, no behavioral measurement of this time scale exists. To fill this gap, we introduce a method based on a statistical model for the effect of the previous trial on the current trial (i.e., CSE) and extend this to the effects of more remote trials. Briefly, the trial-by-trial adaptation captured by the

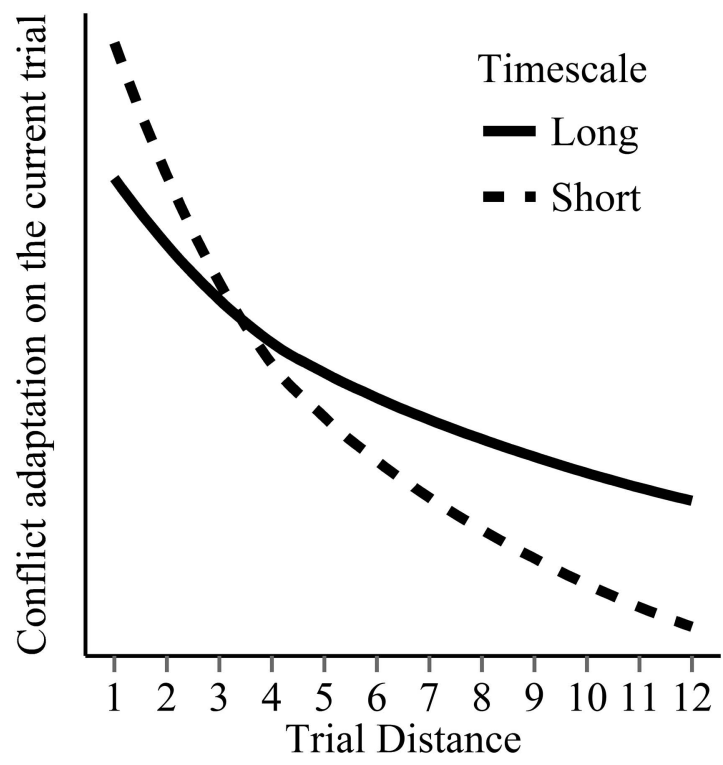

Figure 1. Hypothetical influence of previous conflict on the current trial. The plot shows conflict adaptation (i.e., the interaction between current and previous congruency) as a function of trial distance. For example, when trial distance is 5 , the corresponding value on the $y$-axis represents the interaction between congruency on the current trial and congruency on the fifth trial back (i.e., $C_{i} \times C_{i-5}$ ). The dashed curve represents a situation where the current trial is mainly affected by the most recent trials (i.e., trials with a small distance to the current trial). The steep slope of this curve indicates that effects of previous trials quickly decline with increasing trial distance, which reflects a small window of control. This implies that control is applied over a short time scale. The solid curve represents a situation where, compared to the dashed curve, performance on the current trial is less affected by recent trials and more by remote trials. The gradual decline of this curve indicates a large window, or the exertion of control over a longer time scale. 
CSE is reflected in the interaction between current and previous congruency, $C_{i} \times C_{i-1}$, where variable $C_{i}$ indicates congruency status $(0=$ congruent, $1=$ incongruent) on trial $i$ (Notebaert $\&$ Verguts, 2007). In our method, regressors are added to this statistical model to capture the interaction between current congruency and congruency on more remote trials $\left(C_{i} \times C_{i-2}, C_{i} \times C_{i-3}\right.$, etc.). We then assess how strongly the effects of previous trials decrease as a function of trial distance (see Figure 1). In this way, the degree to which previous trials affect the current trial as a function of trial distance can be computed.

Inspiration for this method was found in the reinforcement learning literature (e.g., Behrens, Woolrich, Walton, \& Rushworth, 2007; Bromberg-Martin, Matsumoto, Nakahara, \& Hikosaka, 2010; Silvetti, Seurinck, van Bochove, \& Verguts, 2013). Here, models predict reward learning rates based on the outcome of previous trials. The learning rate can be conceptualized as a "window size," or the number of previous trials that is taken into account. The smaller the window size, the fewer trials in the past are used to predict current reward. In a similar vein, window size can be used as a measure for the time scale of control. In this case, a small window (steep curve in Figure 1, dashed line) entails that performance on the current trial is mostly affected by conflict on the preceding trial and less by more remote trials. This is indicative of a transient control mode operating on a short time scale. A large window (flatter curve in Figure 1, solid line) entails that performance on the current trial is also affected by conflict on more remote trials. This is indicative of a more sustained control mode operating over a longer time scale.

The use of window size as a measure of the time scale of cognitive control builds on the assumption that the CSE is an indicator of cognitive control. This interpretation of the CSE is not without controversy. Alternatively, it has been claimed that the CSE is due to associative mechanisms driven by the repetition or integration of stimulus or response features (i.e., repetition account; Hommel, Proctor, \& Vu, 2004; Mayr, Awh, \& Laurey, 2003). Current consensus however seems to be that the CSE embodies both stimulus-specific repetitions and cognitive control mechanisms (for reviews, see Duthoo et al., 2014; Egner, 2007) and it has been proposed that cognitive control is in fact built on repetitions of stimulus, response, and control features (Abrahamse, Braem, Notebaert, \& Verguts, 2016; Verguts \& Notebaert, 2009). Moreover, in the current study we investigate differences in time scales between conditions with varying proportion congruency and volatility. While natural from a cognitive control perspective, there seems to be no a priori reason why repetition accounts would predict differences in time scales between these conditions. Hence, our method may also inform this discussion in the literature.

We applied our method using a flanker task with four different blocks with varying proportions of congruent and incongruent items: (a) a neutral block with 50\% congruent items; (b) an MC block with $80 \%$ congruent items; (c) an MI block with $20 \%$ congruent items; and (d) a volatile block, where the proportion congruency changed every 20 trials. We hypothesized that in MC blocks, control would operate on a short time scale, reflected in fast adjustments in control and hence a small window. In MI blocks, slower updating and thus a larger window was expected, indicating control operating over a longer time scale (De Pisapia \& Braver, 2006). A volatile block was added because it has been suggested that volatility increases learning rates (Behrens et al.,
2007; Jiang et al., 2014) and hence may also elicit shorter time scales of control.

\section{Method}

\section{Participants}

Forty-five participants were recruited from the participant pool at the Vrije Universiteit Brussel. All participants gave written informed consent and received a course credit or monetary reward for participation. Participants with overall error rates larger than $25 \%$ were excluded $(n=3)$, leaving 42 participants included for analysis (32 female, mean age $=22.29 \pm 5.77)$.

\section{Material and Procedure}

An arrow flanker task was used consisting of a central target arrow flanked by two distractor arrows on both sides. Congruent (i.e., $<<<<<$ and $>>>>>$ ) and incongruent (e.g., $<<><<$ and $>><>>$ ) flanker stimuli ( $4^{\circ}$ wide and $1^{\circ}$ high) were presented in white against a black background in the center of the screen. Participants were instructed to press a left or right button on a response box (Cedrus RB-830; Cedrus Corporation, San Pedro, CA) with their corresponding index finger as fast and as accurately as possible in response to the central target arrow. Each trial started with a $500-\mathrm{ms}$ fixation cross, followed by a $500-\mathrm{ms}$ blank screen. This was followed by the flanker stimulus, which remained on the screen until the participant responded. No feedback was given.

Four different blocks of 160 trials each were created with different proportions of congruent and incongruent trials. In the neutral, MC, and MI block, the ratio congruent:incongruent trials was 50:50, 80:20, and 20:80, respectively. In the volatile block, the overall ratio was 50:50, but the ratio changed every 20 trials between 80:20 and 20:80. Presentation of the trials in each block was random. Each participant completed all block types; block order was counterbalanced across participants using a Latin square. Blocks were separated by a 60 -s pause.

Before the experiment, we explained to participants that there were easy (i.e., congruent) and difficult (i.e., incongruent) trials, and blocks with mainly easy, mainly difficult, or an equal number of easy and difficult trials. Participants were asked after each block whether they thought the previous block contained mainly easy trials, an equal number of easy and difficult trials, or mainly difficult trials. Answers were given on a scale from 1 to $3(1=$ mainly easy, 3 = mainly difficult). Prior to the main experiment, participants completed 16 practice trials (50\% congruent) with accuracy feedback, followed by a practice question about the ratio of easy and difficult trials.

\section{Analysis}

The following trials were excluded: the first trial of each block $(0.63 \%)$, error trials $(2.79 \%)$, trials following errors $(2.59 \%)$, and trials faster than $250 \mathrm{~ms}(0.03 \%)$ or slower than $1500 \mathrm{~ms}(0.32 \%)$. Next, RTs (in ms) were inverse transformed (1/RT) to better approximate the normal distribution (Kinoshita, Mozer, \& Forster, 2011). Inverse transformed RTs were multiplied by $-10,000$ to restrict the number of decimal places. This way, larger inverse RTs 
reflect slower responses and uninformative decimals are lost, thus facilitating interpretation (Kinoshita et al., 2011).

First, conventional analyses of the PCE and CSE were performed. The mean RTs were entered in a repeated measures analysis of variance (ANOVA) with Block Type (neutral, MC, MI, or volatile), Current-Trial Congruency (congruent or incongruent), and Previous-Trial Congruency (congruent or incongruent) as within-subjects factors. A Greenhouse-Geisser correction was applied when the assumption of sphericity was violated.

Second, for the time scale analyses, the RTs were analyzed at two consecutive (and hierarchically related) levels. At the first level, multiple regression was performed with RT on each separate trial as dependent variable. The regression was performed separately for each block of trials within every participant (R and SPSS code available in Supplemental Material). The predictors included in the model were congruency of the current trial $\left(C_{i}\right)$, congruency of the $k$ preceding trials $\left(C_{i-k}\right.$ where $k$ represents the trial distance from the current trial $i$ ), and the interactions between current-trial congruency and preceding-trial congruency $\left(C_{i} C_{i-k}\right)$. All available trials were included, implying that the number of trials included to estimate each regressor depended on the trial distance that the regressor was associated to. For example, for regressors with Trial Distance 4 (i.e., $C_{i-4}$ and $C_{i} C_{i-4}$ ), no data is available for the first four trials of each block, as only trials with at least four preceding trials can be included for these regressors. This resulted in the statistical model

$$
\begin{aligned}
R T= & \beta_{0}+\beta_{1} C_{i}+\ldots+\beta_{K+1} C_{i-K}+\beta_{K+2} C_{i} C_{i-1}+\ldots \\
& +\beta_{2 K+1} C_{i} C_{i-K},
\end{aligned}
$$

when we modeled influences until $K$ trials back. Each variable $C_{i}$ could take on the value of 0 (congruent) or 1 (incongruent). Of key interest were the effects of previous trials on the current trial, reflected in the interaction terms $C_{i} C_{i-k}$. These interaction terms capture the degree to which congruency on trial $i-k$ affects adaptation on the current trial $(i)$. It can be considered an extension of the CSE from one trial into the past to $K$ trials into the past (while controlling for the other variables in the model). First, we performed the regressions per block type within each participant with $K=14$ to investigate how far "back in the past" previous trials had an effect on the current trial (one-sample $t$ tests). Because this turned out to be the case until 12 trials back, we fitted a model with $K=12$ and used this model subsequently.

At the second level, the 12 regression coefficients of the interaction terms $\left(C_{i} C_{i-k}, k=1 \ldots 12\right)$ estimated by the model of Level 1 for each block type and participant were entered as dependent variables in a linear mixed model with the continuous variable trial distance (1-12) and the factor Block Type (neutral, MC, MI, or volatile) as fixed predictors. We will refer to this second-level dependent variable $\left(C_{i} C_{i-k}\right)$ as the conflict adaptation weight. The conflict adaptation weight reflects the adjustment in CE depending on congruency on previous trials. For example, with Trial Distance 1 , the conflict adaptation weight (i.e., $C_{i} C_{i-1}$ ) corresponds to the standard CSE, or the adjustment of the CE based on congruency of the directly preceding trial (while controlling for the other factors in the model). A negative conflict adaptation weight for a distance of 1 then indicates that this $\mathrm{CE}$ is smaller when the preceding trial was incongruent (i.e., the standard CSE), whereas a positive conflict adaptation weight indicates that this $\mathrm{CE}$ is larger when the preceding trial was incongruent (i.e., a reversed CSE). When the trial distance is 2 , a negative conflict adaptation weight indicates that the $\mathrm{CE}$ is smaller when the trial two trials ago was incongruent.

In the second-level analysis, the predictor trial distance was log transformed (log trial distance) to improve fit to the nonlinear relation between trial distance and conflict adaptation weight. This predictor was subsequently mean-centered to allow interpretation of the intercept (i.e., the intercept then reflects the conflict adaptation weight of the average log trial distance). For the secondlevel block type predictor, the neutral block was taken as the reference. The linear mixed model was extended stepwise and each model was compared to its initial model to test for significance of the added predictor. Akaike information criterion (Akaike, 1974) is reported as a measure of model fit with lower values indicating a better fit. This measure penalizes model complexity and discourages overfitting, which implies that more complex models are only favored if they explain the data substantially better than the simpler model. Models were statistically compared using the likelihood ratio $\left(\chi^{2}\right)$. It has been proposed to keep the random effects structure maximal (Barr, Levy, Scheepers, \& Tily, 2013). However, it has also been argued that this often results in overparameterized models that fail to converge (Bates, Kliegl, Vasishth, \& Baayen, 2015), which was also the case when we fitted maximal models. Therefore, random effects were chosen such that convergence was obtained for each model in every step, which resulted in only including a random intercept for participant. All models were fitted using maximum likelihood in the lme 4 package (Bates, Maechler, Bolker, \& Walker, 2015) for R (R Core Team, 2015).

\section{Results}

\section{Conventional Analysis of PCE and CSE}

A repeated-measures ANOVA was conducted with the withinsubject factors Block Type (neutral, MC, MI, or volatile), Congruency (congruent or incongruent), and Previous-Trial Congruency (congruent or incongruent). A main effect of block type was found, $F(2.39,97.98)=3.78, p=.020$, which was due to slower RTs in the volatile compared to the MC block, $t(41)=2.86, p=$ .038. Main effects of congruency, $F(1,41)=657.04, p<.001$, and previous-trial congruency, $F(1,41)=9.07, p=.004$, were also observed, indicating slower responses when the current trial was incongruent (i.e., a CE), or the previous trial was incongruent (i.e., postconflict slowing), respectively.

Block type and congruency interacted significantly, $F(2.30$, $94.41)=43.57, p<.001$, indicating a PCE. To study this effect in more detail, CEs were computed and compared between blocks using paired sample $t$ tests (see Figure 2A). This revealed that the CE was smallest in the MI block and increased incrementally over the neutral, volatile, and MC block. More specifically, a smaller CE was observed in the MI block compared to all other blocks, respectively, neutral block, $t(41)=5.22, p<.001$; volatile block, $t(41)=8.25, p<.001$; MC block, $t(41)=12.64, p<.001$. A smaller CE was also found in the neutral block compared to the volatile block, $t(41)=4.19, p<.001$, and MC block, $t(41)=$ 13.23, $p<.001$. The CE was also smaller in the volatile block compared to the MC block, $t(41)=10.52, p<.001$. 
A Conventional analyses
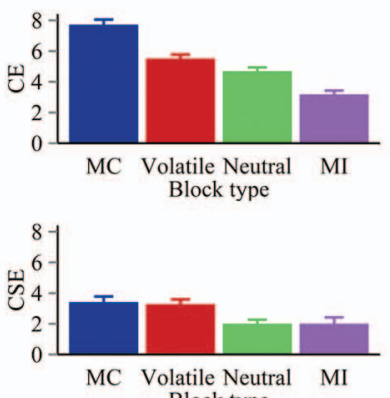

Block type

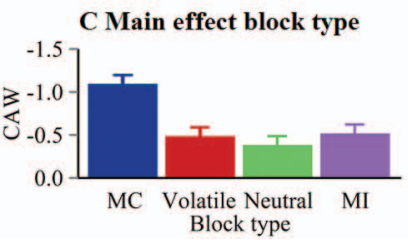

B Time scale of control

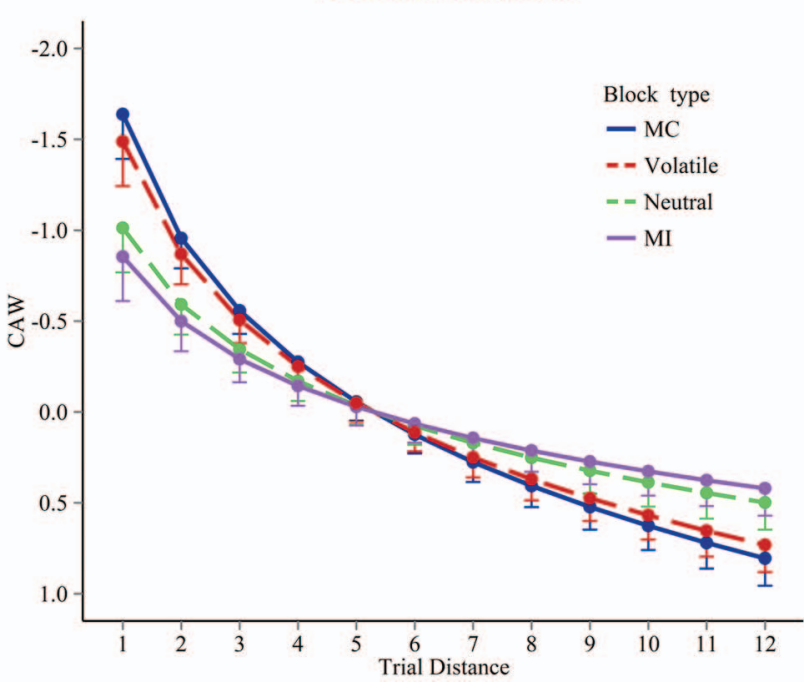

Figure 2. Results of conventional and time scale analyses. (A) Congruency effects (CEs) and congruency sequence effects (CSEs) per block type, expressed in mean inverse transformed response times (i.e., $-10,000 /$ RT). (B) Model estimates (each pair of points connected by piecewise linearly interpolated lines) of Model 4. Each condition is plotted after subtracting its intercept. The original scale of trial distance is displayed on the $x$-axis, not log trial distance (which was included in the linear mixed models for statistical testing). This way, the nonlinear relationship between trial distance and conflict adaptation weight is maintained in the figure. (C) Main effect of block type (i.e., the conflict adaptation weight of the average log trial distance). Error bars represent standard errors of the mean $(\mathrm{A}, \mathrm{C})$ or of the model prediction (B). CAW = conflict adaptation weight; $\mathrm{MC}=$ mainly congruent; $\mathrm{MI}=$ mainly incongruent. See the online article for the color version of this figure

No two-way interaction was found between block type and previous-trial congruency, $F(2.49,102.06)=0.93, p=.43$, but congruency and previous-trial congruency did interact, $F(1,41)=$ $138.10, p<.001$, pointing at a smaller $\mathrm{CE}$ after an incongruent trial than after a congruent trial, or a CSE. This CSE was modulated by block type, as evident in the three-way interaction between block type, congruency, and previous-trial congruency, $F(2.56,105.12)=6.96, p<.001$. To specify this three-way interaction, CSEs were computed and compared between blocks using paired sample $t$ tests (see Figure 2A). A smaller CSE was found in the MI block compared to the volatile block, $t(41)=2.81$, $p=.007$, and the MC block, $t(41)=3.23, p=.002$. The CSE was also smaller in the neutral block compared to the volatile block, $t(41)=4.12 p<.001$, and the MC block, $t(41)=3.74, p<.001$. The other comparisons revealed no significant effects (both $p \mathrm{~s}>$ .74). One-sample $t$ tests indicated that, despite these differences between blocks, a significant CSE was obtained in every block (all $p \mathrm{~s}<.001)$.

\section{The Time Scale of Control}

One-sample $t$ tests were conducted on the beta weights for the respective interaction terms (i.e., $C_{i} C_{i-k}$, averaged across blocks and hence orthogonal to our hypothesis) to asses significance of each regressor's contribution to the model. Figure 3 displays the results of these analyses for maximal trial distance $K=14$. As evident from the figure, all trial distances up to 12 , except for a distance of 9, had a significant or marginally significant contribution. Contrarily, the regression coefficients for Trial Distances 13 and 14 did not differ from 0 . Hence, regressors up to Trial Distance 12 were included in the Level 1 model, resulting in the model

$$
\begin{aligned}
R T= & \beta_{0}+\beta_{1} C_{i}+\beta_{2} C_{i-1}+\ldots+\beta_{13} C_{i-12}+\beta_{14} C_{i} C_{i-1}+\ldots \\
& +\beta_{25} C_{i} C_{i-12} .
\end{aligned}
$$

Table 1 displays the fitted statistical models and comparisons of each model relative to the prior one. Results show that the model with a main effect of log trial distance (Model 1) explained the data better than the null model with only an intercept, $\chi^{2}(1)=$

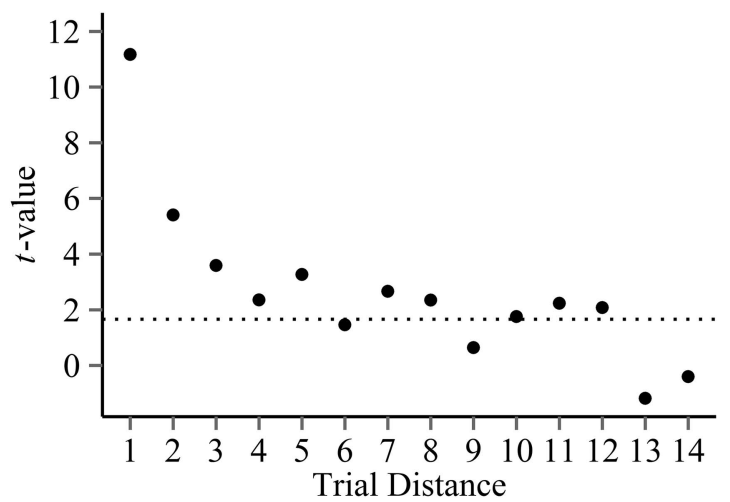

Figure 3. One-sample $t$ values for the interaction terms of the model of Level $1(K=14)$, plotted as a function of trial distance. The dotted line indicates the critical $t$ value $(t=1.66)$ for a one-sided, one-sample $t$ test with $d f=167$. 
Table 1

Model Comparisons $(K=12)$

\begin{tabular}{lrrrrrr}
\hline \multicolumn{1}{c}{ Model } & $d f$ & AIC & log lik. & Test & \multicolumn{1}{c}{$\chi^{2}$} & $p$ \\
\hline 0. (Intercept) & 3 & 9,016 & $-4,505$ & & & \\
1. Log trial distance & 4 & 8,998 & $-4,445$ & 1 vs. 0 & 119.85 & $<.001$ \\
2. Block type & 6 & 8,991 & $-4,490$ & 2 vs. 0 & 30.55 & $<.001$ \\
3. Log trial distance + block type & 7 & 8,871 & $-4,429$ & 3 vs. 1 & 32.64 & $<.001$ \\
& & & & 3 vs. 2 & 121.94 & $<.001$ \\
4. Log trial distance + block type + & 10 & 8,869 & $-4,424$ & 4 vs. 3 & 8.48 & .037 \\
log trial distance $\times$ block type & 10 & & & &
\end{tabular}

Note. $\quad$ AIC $=$ Akaike information criterion; $\log$ lik. $=\log$ likelihood.

$119.85, p<.001$. A model with a main effect of block type (Model 2) also explained the data better than the null model, $\chi^{2}(3)=30.55$ $p<.001$. Entering both main effects in one model (Model 3) resulted in a better model fit than a model with only log trial distance, Model $1, \chi^{2}(3)=32.64, p<.001$, or only block type, Model $2, \chi^{2}(1)=121.94, p<.001$. Allowing the main effects to interact (Model 4) further improved model fit compared to Model $3, \chi^{2}(3)=8.48, p=.037$. The regression coefficients of the optimal Model 4 are displayed in Table 2. Figure 2B displays the estimates of Model 4, corrected for the intercept of each condition.

To check if the conflict adaptation weight varied with trial distance for every block type, a model with log trial distance was compared to a model without log trial distance (i.e., the null model) for each block type separately. This revealed that trial distance indeed predicted the conflict adaptation weight in each block (all $p$ s $<.01)$.

Planned linear contrasts (one-sided) were computed for pairwise comparisons of the slopes (i.e., time scales) of the four conditions, estimated by Model 4. Because the neutral block was set as the reference level, the slope of this condition is reflected in the beta for $\log$ trial distance. The slopes of the other three conditions are the sums of the betas for log trial distance and each interaction term (see Table 2). For example, the slope of the MC block equals the slope for $\log$ trial distance plus the beta for log trial distance $\times$ Block MC. Pairwise comparisons revealed a smaller slope for the MI block ( $\beta=0.37$ ) compared to the volatile block $(\beta=0.65)$, $z=2.02, p=.022$, and the MC block $(\beta=0.72), z=2.49, p=$ .006 , but not compared to the neutral block $(\beta=0.44), z=0.50$, $p=.33$. The slope in the neutral block was also smaller than the

Table 2

Coefficient Estimates ( $\beta s)$ of Linear Mixed Model 4 With Conflict Adaptation Weight Predicted by Log Trial Distance, Block Type (Neutral, MC, MI, Volatile), and Their Interaction

\begin{tabular}{lrr}
\hline \multicolumn{1}{c}{ Variable } & \multicolumn{1}{c}{$\beta(S E)$} & \multicolumn{1}{c}{$t$} \\
\hline (Intercept) & $-.38(.10)$ & -3.74 \\
Log trial distance & $.44(.10)$ & 4.56 \\
Block MC & $-.71(.14)$ & -5.20 \\
Block volatile & $-.14(.14)$ & -1.00 \\
Block MI & $-.10(.14)$ & -.75 \\
Log trial distance $\times$ block MC & $.27(.14)$ & 1.99 \\
Log trial distance $\times$ block volatile & $.21(.14)$ & 1.51 \\
Log trial distance $\times$ block MI & $-.07(.14)$ & -.50 \\
\hline
\end{tabular}

Note. $\mathrm{MC}=$ mainly congruent; $\mathrm{MI}=$ mainly incongruent. Neutral block type served as reference level. slope of the MC block, $z=1.99, p=.023$. A trend toward a smaller slope for the neutral block compared to the volatile block was found, $z=1.51, p=.065$. The volatile and MC block did not differ in slope, $z=0.48, p=.32$. These differences in slopes are shown in Figure 2B.

Linear contrasts were also computed for pairwise comparisons of the main effect of each block type (i.e., the conflict adaptation weight of the average log trial distance), estimated by Model 4 (see Figure $2 \mathrm{C}$ ). Here, the main effects for the MC, MI, and volatile block are computed by adding the main effect of the neutral block (i.e., the intercept) to the main effect of the MC, MI, or volatile block (see Table 2). Compared to the MC block $(-1.09)$, the main effects were smaller (i.e., less negative) in the volatile block $(-0.52), z=4.20, p<.001$, the neutral block $(-0.38), z=5.19$, $p<.001$, and the MI block $(-0.49), z=4.45, p<.001$. No other contrasts for the main effect of block were significant (all $p \mathrm{~s}>$ $.32)$.

\section{Proportion Congruency Awareness}

Finally, we checked whether participants were aware of the differences in proportion congruency between blocks. ${ }^{1}$ Friedman's ANOVA revealed that this was indeed the case, $\chi^{2}(3)=66.74$, $p<.001$. Post hoc Wilcoxon signed-ranks test showed that the median rating for the MI block (median rating = 3) was higher than the median ratings for the neutral block (median rating $=2$ ), $Z=3.56, p<.001$, the volatile block (median rating $=2$ ), $Z=$ $4.26, p<.001$, and the MC block (median rating $=1$ ), $Z=5.07$, $p<.001$. Furthermore, the median rating for the neutral and volatile blocks were higher compared to the MC block, $Z=5.06$, $p<.001$, and $Z=4.56, p<.001$, respectively. No difference in rating was found between the neutral and volatile block, $Z=0.23$, $p=.82$.

\section{Discussion}

We introduced a novel method to quantify temporal variations in control, by extending the CSE to more remote trials and computing the effects of previous trials on the current trial as a function of trial distance. The rate at which these effects decrease with trial distance was taken as a measure of the time scale of control, with a more gradual decline reflecting a longer time scale.

\footnotetext{
${ }^{1}$ These analyses were performed on 34 participants because the rating scores of eight participants were missing due to a technical failure.
} 
We observed a longer time scale in the MI block than in the MC block, suggesting an influence from more (distant) trials when conflict is frequent. This is in agreement with models assuming that cognitive control is relatively more affected by remote events when conflict is frequent (i.e., sustained control) and relatively more by recent events when conflict is infrequent (i.e., transient control; Botvinick et al., 2001; De Pisapia \& Braver, 2006). These models generally explain differences in control modes by adjustments in time scale parameters, implying that control becomes sustained when conflict is frequent, leaving less room for trial-bytrial variations. The method we introduced here provides a direct empirical measure of this dissociation.

Compared to the MC block, the neutral block also showed a longer time scale of control, but no difference was found between the neutral and MI block. These findings suggest that differences in control modes between MC and MI blocks are due to a switch to a shorter time scale in the MC block rather than to a longer time scale in the MI block. This is in contrast with the suggestion that cognitive control over short time scales is the default mode (Braver, Gray, \& Burgess, 2007). However, it is in agreement with the finding that participants decrease their control in response to a cue that indicates that the next block will be MC but do not increase their control when cued with an upcoming MI block (Bugg et al., 2015). Furthermore, some authors have claimed that the relaxation of control after a congruent trial drives the CSE more than the strengthening of control after an incongruent trial (Lamers \& Roelofs, 2011; Schlaghecken \& Martini, 2011), which also suggests that at least for some tasks, sustained control is the default mode which is abandoned in situations where congruent trials dominate and conflict is rare.

A smaller time scale was also found for volatile compared to MI blocks. This can be explained by a lower conflict frequency in the volatile (i.e., 50\%) than in the MI block (i.e., 80\%). A trend toward a smaller time scale in the volatile block compared to the neutral block was also present. The volatile block is identical to the neutral block with regard to block-wide conflict frequency, as also confirmed by the similar subjective evaluation of both blocks. However, the changing proportion congruency creates an instable environment which may cause a bias toward more recent information over remote information (Behrens et al., 2007; Jiang et al., 2014).

With regard to the conventional analyses, the overall pattern of CSEs mirrors that of the time scales. Larger CSEs in MC and volatile blocks compared to MI and neutral blocks were found, matching the shorter time scales in $\mathrm{MC}$ and volatile blocks. Although this CSE pattern suggests that trial-by-trial adaptations are smaller when conflict is frequent or the environment is predictable, it does not reveal how conflict on recent and remote trials is weighted to influence current conflict processing. For example, there may be conditions where the effect of recent conflict on the current trial is similar (i.e., equal CSEs between conditions), yet differences emerge in the effects of more remote trials. Our time scale approach confirms that there is indeed a larger effect of recent trials in $\mathrm{MC}$ and neutral blocks but highlights that this effect decays rapidly with increasing trial distance, resulting in smaller time scales of control.

Other studies that went beyond the CSE have shown before that more distant trials influence control on the current trial. Control is generally increased when the current trial is preceded by a series of repeated incongruent trials (Horga et al., 2011; Jiménez \& Mén- dez, 2013). An important difference with the method introduced here is that these studies did not directly compute the effect of previous trials on the current trial but instead computed the accumulated effect of incongruency on the three or four previous trials. The current method is more fine-grained, and also more powerful because it exploits more data. This makes it more suitable and sensitive to explore differences between conditions.

It has been argued that series of (in)congruent trials create expectation and that these expectations drive adaptation on the current trial (Duthoo, Wühr, \& Notebaert, 2013). For example, when incongruent trial repetitions are frequent, as is the case in MI blocks, participants might expect another incongruent trial and prepare control accordingly. Although this is in agreement with the original interpretation of the CSE (Gratton et al., 1992), the exact contribution of expectancies to cognitive control is an unresolved issue (Jiménez \& Méndez, 2013). Regardless which exact mechanisms drive sequential adjustments in control - expectancies, automatic adaptation, or perhaps yet another process - the main point of the current study is that longer-time-scale effects can be statistically modeled and can indeed be observed.

Another open question is how longer-time-scale control is sustained exactly within and between trials. One possibility is that control is steadily sustained throughout the block both at trial presentation and in the intervals between trials. Alternatively, control could also be routinely activated just in time at or before trial presentation and relaxed between trials. These two mechanisms are hard to differentiate, because control is only measured at trial presentation in most experiments. Instead, a measure in the intertrial intervals would be needed. This could for example be achieved by unexpectedly presenting trials within the intertrial interval or by measuring neurophysiological markers of cognitive control such as electroencephalographic theta power (Cavanagh \& Frank, 2014). Regardless of which mechanism is active, fluctuations in control at the moment of trial presentation are minimal in both scenarios and in that sense control is stable over trials.

One possible limitation of the method we introduced is that the Level 1 regression models included regressors for current and previous congruency and their interactions, but not for interactions between intermediate trials (e.g., between $C_{i-1}$ and $C_{i-2}$ ) or higher-order interactions involving several preceding trials. A practical reason for this restriction is to prevent overfitting the regression models (e.g., adding all first order interactions would expand the Level 1 model with 66 regressors). However, excluding these terms implies that the observed time scale differences can only be interpreted independently from intermediate interactions. Still, it is possible that the effect of congruency on Trial $i-k$ on Trial $i$ is moderated by the intermediate trials instead of affecting Trial $i$ directly. Future research could investigate this issue, but as a consequence of the quickly increasing number of parameters, will not be able to look "back in time" as far as in the current study.

Apart from our proportion congruency and volatility manipulations, several other factors are likely to elicit different time scales of cognitive control. One factor (or individual difference) that has often be linked to differences in control mode is working memory capacity (e.g., Gulbinaite \& Johnson, 2014; Meier \& Kane, 2013; Unsworth, Redick, Spillers, \& Brewer, 2012). Also, clinical conditions known to affect cognitive control, such as attentional disorders (Nigg, 2005) and schizophrenia (Lesh, Niendam, Minzenberg, \& Carter, 2011), may exhibit difficulties with adopting 
appropriate time scales in different contexts. It has also been shown that in healthy populations, both children and older adults have difficulties coordinating optimal control strategies (Chevalier, 2015; Jimura et al., 2010; Paxton, Barch, Racine, \& Braver, 2008), and that task-strategy training can reduce this bias (Braver, Paxton, Locke, \& Barch, 2009; Paxton, Barch, Storandt, \& Braver, 2006). Quantifying the time scale of control may allow more straightforward evaluation of the factors that underlie these difficulties and the time scale may also serve as a target for cognitive training. Future research could also address whether explicitly instructing participants to focus on the conflict frequency, as was the case in the current study, influences time scale measures. Finally, the influence of associative processes and strategy learning on the time-scale of control also remains an open question, which could be addressed by increasing the number of stimuli and using more complex stimulus-response mappings.

In conclusion, we have successfully applied a novel method to identify the time scale of cognitive control. Shorter time scales of control were demonstrated when conflict was rare and when the context was volatile. This is in line with theories assuming transient control (i.e., control over a shorter time scale) in rare-conflict and volatile conditions, and sustained control (i.e., control over longer time scales) when conflict is more frequent. The method offers promising and straightforward opportunities to quantify variations in the time scale of control between individuals and conditions.

\section{References}

Abrahamse, E., Braem, S., Notebaert, W., \& Verguts, T. (2016). Grounding cognitive control in associative learning. Psychological Bulletin, 142, 693-728. http://dx.doi.org/10.1037/bul0000047

Akaike, H. (1974). A new look at the statistical model identification. IEEE Transactions on Automatic Control, 19, 716-723. http://dx.doi.org/10 .1109/TAC.1974.1100705

Appelbaum, L. G., Boehler, C. N., Davis, L. A., Won, R. J., \& Woldorff, M. G. (2014). The dynamics of proactive and reactive cognitive control processes in the human brain. Journal of Cognitive Neuroscience, 26, 1021-1038. http://dx.doi.org/10.1162/jocn_a 00542

Barr, D. J., Levy, R., Scheepers, C., \& Tily, H. J. (2013). Random effects structure for confirmatory hypothesis testing: Keep it maximal. Journal of Memory and Language, 68, 255-278. http://dx.doi.org/10.1016/j.jml .2012 .11 .001

Bates, D., Kliegl, R., Vasishth, S., \& Baayen, H. (2015). Parsimonious Mixed Models. arXiv:1506.04967 [stat.ME], 1-27. Retrieved from http:// arxiv.org/abs/1506.04967

Bates, D., Maechler, M., Bolker, B., \& Walker, S. (2015). Fitting linear mixed-effects models using lme4. Journal of Statistical Software, 67, 1-48. http://dx.doi.org/10.18637/jss.v067.i01

Behrens, T. E. J., Woolrich, M. W., Walton, M. E., \& Rushworth, M. F. S. (2007). Learning the value of information in an uncertain world. Nature Neuroscience, 10, 1214-1221. http://dx.doi.org/10.1038/nn1954

Botvinick, M. M., Braver, T. S., Barch, D. M., Carter, C. S., \& Cohen, J. D. (2001). Conflict monitoring and cognitive control. Psychological Review, 108, 624-652. http://dx.doi.org/10.1037/0033-295X.108.3.624

Braver, T. S. (2012). The variable nature of cognitive control: A dual mechanisms framework. Trends in Cognitive Sciences, 16, 106-113. http://dx.doi.org/10.1016/j.tics.2011.12.010

Braver, T. S., Gray, J. R., \& Burgess, G. C. (2007). Explaining the many varieties of working memory variation: Dual mechanisms of cognitive control. In A. A. R. Conway, C. Jarrold, M. J. Kane, A. Miyake, \& J. N. Towse (Eds.), Variation in working memory (pp. 1-79). Oxford, United Kingdom: Oxford University Press.

Braver, T. S., Paxton, J. L., Locke, H. S., \& Barch, D. M. (2009). Flexible neural mechanisms of cognitive control within human prefrontal cortex. Proceedings of the National Academy of Sciences, 106, 7351-7356. http://dx.doi.org/10.1073/pnas.0808187106

Bromberg-Martin, E. S., Matsumoto, M., Nakahara, H., \& Hikosaka, O. (2010). Multiple timescales of memory in lateral habenula and dopamine neurons. Neuron, 67, 499-510. http://dx.doi.org/10.1016/j.neuron.2010 .06 .031

Bugg, J. M., \& Chanani, S. (2011). List-wide control is not entirely elusive: Evidence from picture-word Stroop. Psychonomic Bulletin \& Review, 18, 930-936. http://dx.doi.org/10.3758/s13423-011-0112-y

Bugg, J. M., \& Crump, M. J. C. (2012). In support of a distinction between voluntary and stimulus-driven control: A review of the literature on proportion congruent effects. Frontiers in Psychology, 3, 367.

Bugg, J. M., Diede, N. T., Cohen-Shikora, E. R., \& Selmeczy, D. (2015). Expectations and experience: Dissociable bases for cognitive control? Journal of Experimental Psychology: Learning, Memory, and Cognition, 41, 1349-1373. http://dx.doi.org/10.1037/xlm0000106

Bugg, J. M., McDaniel, M. A., Scullin, M. K., \& Braver, T. S. (2011). Revealing list-level control in the Stroop task by uncovering its benefits and a cost. Journal of Experimental Psychology: Human Perception and Performance, 37, 1595-1606. http://dx.doi.org/10.1037/a0024670

Cavanagh, J. F., \& Frank, M. J. (2014). Frontal theta as a mechanism for cognitive control. Trends in Cognitive Sciences, 18, 414-421. http://dx doi.org/10.1016/j.tics.2014.04.012

Chevalier, N. (2015). The development of executive function: Toward more optimal coordination of control with age. Child Development Perspectives, 9, 239-244. http://dx.doi.org/10.1111/cdep.12138

De Pisapia, N., \& Braver, T. S. (2006). A model of dual control mechanisms through anterior cingulate and prefrontal cortex interactions. Neurocomputing, 69, 1322-1326. http://dx.doi.org/10.1016/j.neucom.2005 .12 .100

Duthoo, W., Abrahamse, E. L., Braem, S., Boehler, C. N., \& Notebaert, W (2014). The heterogeneous world of congruency sequence effects: An update. Frontiers in Psychology, 5, 1001.

Duthoo, W., Wühr, P., \& Notebaert, W. (2013). The hot-hand fallacy in cognitive control: Repetition expectancy modulates the congruency sequence effect. Psychonomic Bulletin \& Review, 20, 798-805. http://dx .doi.org/10.3758/s13423-013-0390-7

Egner, T. (2007). Congruency sequence effects and cognitive control. Cognitive, Affective \& Behavioral Neuroscience, 7, 380-390. http://dx doi.org/10.3758/CABN.7.4.380

Entel, O., Tzelgov, J., \& Bereby-Meyer, Y. (2014). Proportion congruency effects: Instructions may be enough. Frontiers in Psychology, 5, 1108.

Funes, M. J., Lupiáñez, J., \& Humphreys, G. (2010). Sustained vs. transient cognitive control: Evidence of a behavioral dissociation. Cognition, 114, 338-347. http://dx.doi.org/10.1016/j.cognition.2009.10.007

Gratton, G., Coles, M. G. H., \& Donchin, E. (1992). Optimizing the use of information: Strategic control of activation of responses. Journal of Experimental Psychology: General, 121, 480-506. http://dx.doi.org/10 .1037/0096-3445.121.4.480

Gulbinaite, R., \& Johnson, A. (2014). Working memory capacity predicts conflict-task performance. Quarterly Journal of Experimental Psychology: Human Experimental Psychology, 67, 1383-1400. http://dx.do .org/10.1080/17470218.2013.863374

Hommel, B., Proctor, R. W., \& Vu, K.-P. L. (2004). A feature-integration account of sequential effects in the Simon task. Psychological Research, 68, 1-17. http://dx.doi.org/10.1007/s00426-003-0132-y

Horga, G., Maia, T. V., Wang, P., Wang, Z., Marsh, R., \& Peterson, B. S. (2011). Adaptation to conflict via context-driven anticipatory signals in 
the dorsomedial prefrontal cortex. The Journal of Neuroscience, 31, 16208-16216. http://dx.doi.org/10.1523/JNEUROSCI.2783-11.2011

Jacoby, L. L., Lindsay, D. S., \& Hessels, S. (2003). Item-specific control of automatic processes: Stroop process dissociations. Psychonomic Bulletin \& Review, 10, 638-644. http://dx.doi.org/10.3758/BF03196526

Jiang, J., Heller, K., \& Egner, T. (2014). Bayesian modeling of flexible cognitive control. Neuroscience and Biobehavioral Reviews, 46, 30-43. http://dx.doi.org/10.1016/j.neubiorev.2014.06.001

Jiménez, L., \& Méndez, A. (2013). It is not what you expect: Dissociating conflict adaptation from expectancies in a Stroop task. Journal of Experimental Psychology: Human Perception and Performance, 39, 271284. http://dx.doi.org/10.1037/a0027734

Jimura, K., Locke, H. S., \& Braver, T. S. (2010). Prefrontal cortex mediation of cognitive enhancement in rewarding motivational contexts. Proceedings of the National Academy of Sciences of the United States of America, 107, 8871-8876. http://dx.doi.org/10.1073/pnas.1002007107

Kane, M. J., \& Engle, R. W. (2003). Working-memory capacity and the control of attention: The contributions of goal neglect, response competition, and task set to Stroop interference. Journal of Experimental Psychology: General, 132, 47-70. http://dx.doi.org/10.1037/0096-3445 132.1.47

Kinoshita, S., Mozer, M. C., \& Forster, K. I. (2011). Dynamic adaptation to history of trial difficulty explains the effect of congruency proportion on masked priming. Journal of Experimental Psychology: General, 140, 622-636. http://dx.doi.org/10.1037/a0024230

Lamers, M. J. M., \& Roelofs, A. (2011). Attentional control adjustments in Eriksen and Stroop task performance can be independent of response conflict. Quarterly Journal of Experimental Psychology: Human Experimental Psychology, 64, 1056-1081. http://dx.doi.org/10.1080/ 17470218.2010 .523792

Lesh, T. A., Niendam, T. A., Minzenberg, M. J., \& Carter, C. S. (2011). Cognitive control deficits in schizophrenia: Mechanisms and meaning. Neuropsychopharmacology, 36, 316-338. http://dx.doi.org/10.1038/npp .2010 .156

Logan, G. D., \& Zbrodoff, N. J. (1979). When it helps to be misled: Facilitative effects of increasing the frequency of conflicting stimuli in a Stroop-like task. Memory \& Cognition, 7, 166-174. http://dx.doi.org/ 10.3758/BF03197535

Mayr, U., Awh, E., \& Laurey, P. (2003). Conflict adaptation effects in the absence of executive control. Nature Neuroscience, 6, 450-452.

Meier, M. E., \& Kane, M. J. (2013). Working memory capacity and Stroop interference: Global versus local indices of executive control. Journal of Experimental Psychology: Learning, Memory, and Cognition, 39, $748-$ 759. http://dx.doi.org/10.1037/a0029200

Nigbur, R., Schneider, J., Sommer, W., Dimigen, O., \& Stürmer, B. (2015). Ad-hoc and context-dependent adjustments of selective attention in conflict control: An ERP study with visual probes. Neurolmage, 107, 76-84. http://dx.doi.org/10.1016/j.neuroimage.2014.11.052

Nigg, J. T. (2005). Neuropsychologic theory and findings in attentiondeficit/hyperactivity disorder: The state of the field and salient challenges for the coming decade. Biological Psychiatry, 57, 1424-1435. http://dx.doi.org/10.1016/j.biopsych.2004.11.011

Notebaert, W., \& Verguts, T. (2007). Dissociating conflict adaptation from feature integration: A multiple regression approach. Journal of Experimental Psychology: Human Perception and Performance, 33, 12561260. http://dx.doi.org/10.1037/0096-1523.33.5.1256
Paxton, J. L., Barch, D. M., Racine, C. A., \& Braver, T. S. (2008) Cognitive control, goal maintenance, and prefrontal function in healthy aging. Cerebral Cortex, 18, 1010-1028. http://dx.doi.org/10.1093/ cercor/bhm135

Paxton, J. L., Barch, D. M., Storandt, M., \& Braver, T. S. (2006). Effects of environmental support and strategy training on older adults' use of context. Psychology and Aging, 21, 499-509. http://dx.doi.org/10.1037/ 0882-7974.21.3.499

Purmann, S., Badde, S., \& Wendt, M. (2009). Adjustments to recent and frequent conflict reflect two distinguishable mechanisms. Psychonomic Bulletin \& Review, 16, 350-355. http://dx.doi.org/10.3758/PBR.16.2 .350

R Core Team. (2015). R: A language and environment for statistical computing [Computer software]. Vienna, Austria: R Foundation for Statistical Computing. Retrieved from http://www.R-project.org/

Ridderinkhof, K. R. (2002). Micro- and macro-adjustments of task set: Activation and suppression in conflict tasks. Psychological Research, 66, 312-323. http://dx.doi.org/10.1007/s00426-002-0104-7

Scherbaum, S., Fischer, R., Dshemuchadse, M., \& Goschke, T. (2011). The dynamics of cognitive control: Evidence for within-trial conflict adaptation from frequency-tagged EEG. Psychophysiology, 48, 591-600 http://dx.doi.org/10.1111/j.1469-8986.2010.01137.x

Schlaghecken, F., \& Martini, P. (2011). Context, not conflict, drives cognitive control. Journal of Experimental Psychology: Human Perception and Performance, 38, 272-278. http://dx.doi.org/10.1037/a0025791

Schmidt, J. R., \& Besner, D. (2008). The Stroop effect: Why proportion congruent has nothing to do with congruency and everything to do with contingency. Journal of Experimental Psychology: Learning, Memory, and Cognition, 34, 514-523. http://dx.doi.org/10.1037/0278-7393.34.3 .514

Silvetti, M., Seurinck, R., van Bochove, M. E., \& Verguts, T. (2013). The influence of the noradrenergic system on optimal control of neural plasticity. Frontiers in Behavioral Neuroscience, 7, 160.

Stroop, J. R. (1935). Studies of interference in serial verbal reactions. Journal of Experimental Psychology, 18, 643-662. http://dx.doi.org/10 $.1037 / \mathrm{h} 0054651$

Torres-Quesada, M., Funes, M. J., \& Lupiáñez, J. (2013). Dissociating proportion congruent and conflict adaptation effects in a Simon-Stroop procedure. Acta Psychologica, 142, 203-210. http://dx.doi.org/10.1016/ j.actpsy.2012.11.015

Unsworth, N., Redick, T. S., Spillers, G. J., \& Brewer, G. A. (2012). Variation in working memory capacity and cognitive control: Goal maintenance and microadjustments of control. Quarterly Journal of Experimental Psychology: Human Experimental Psychology, 65, 326355. http://dx.doi.org/10.1080/17470218.2011.597865

Verguts, T., \& Notebaert, W. (2008). Hebbian learning of cognitive control: Dealing with specific and nonspecific adaptation. Psychological Review, 115, 518-525. http://dx.doi.org/10.1037/0033-295X.115.2.518

Verguts, T., \& Notebaert, W. (2009). Adaptation by binding: A learning account of cognitive control. Trends in Cognitive Sciences, 13, 252-257. http://dx.doi.org/10.1016/j.tics.2009.02.007

Received February 2, 2016

Revision received June 7, 2016 Accepted September 6, 2016 